\title{
High-Resolution Genotypic Analysis of the Genus Aeromonas by AFLP Fingerprinting
}

\author{
GEERT HUYS, ${ }^{*}$ RENATA COOPMAN, PAUL JANSSEN, AND KAREL KERSTERS \\ Laboratorium voor Microbiologie, Universiteit Gent, B-9000 Ghent, Belgium
}

\begin{abstract}
We investigated the ability of a recently developed genomic fingerprinting technique, named AFLP, to differentiate the 14 currently defined DNA hybridization groups (HGs) in the genus Aeromonas. We also determined the taxonomic positions of the phenospecies Aeromonas allosaccharophila, Aeromonas encheleia, Aeromonas enteropelogenes, and Aeromonas ichthiosmia, which have not been assigned to HGs yet. A total of 98 Aeromonas type and reference strains were included in this study. For the AFLP analysis, the total genomic DNA of each strain was digested with restriction endonucleases ApaI and TaqI. Subsequently, restriction fragments were selectively amplified under high-stringency PCR conditions. The amplification products were electrophoretically separated on a polyacrylamide gel and visualized by autoradiography. Following highresolution densitometric scanning of the resulting band patterns, AFLP data were further processed for a computer-assisted comparison. A numerical analysis of the digitized fingerprints revealed 13 AFLP clusters which, in general, clearly supported the current Aeromonas taxonomy derived from DNA homology data. In addition, our results indicated that there is significant genotypic heterogeneity in Aeromonas eucrenophila (HG6), which may lead to a further subdivision of this species. A. allosaccharophila and $A$. encheleia did not represent a separate AFLP cluster but were found to be genotypically related to $\mathrm{HG8} / 10$ and $\mathrm{HG6}$, respectively. In addition, the results of the AFLP analysis also confirmed the phylogenetic findings that $A$. enteropelogenes and $A$. ichthiosmia are in fact identical to Aeromonas trota (HG13) and Aeromonas veronii (HG8/10), respectively. The results of this study clearly show that the AFLP technique is a valuable new high-resolution genotypic tool for classification of Aeromonas species and also emphasize that this powerful DNA fingerprinting method is important for bacterial taxonomy in general.
\end{abstract}

Members of the genus Aeromonas are facultatively anaerobic, gram-negative rods which have been isolated from various aquatic $(17,39,45)$ and terrestrial $(36,43)$ environments. Some Aeromonas species cause a broad range of extraintestinal infections in cold-blooded animals $(18,40)$, and members of the genus Aeromonas have recently received a great deal of attention because of their role as opportunistic pathogens in human gastroenteritis $(22,27,30)$. Consequently, many microbiologists involved in hospital and public health research have concluded that new techniques for the identification and classification of unknown Aeromonas isolates should be developed. However, data obtained with methods such as sodium dodecyl sulfate-polyacrylamide gel electrophoresis of whole-cell proteins (34), serotyping (16), and pyrolysis mass spectrometry (31) are not consistent with the taxonomic data obtained in conventional biotyping and DNA-DNA homology studies, and it seems that these methods can be used only to discriminate among Aeromonas strains in epidemiological studies. On the other hand, multilocus enzyme electrophoresis and gas-liquid chromatographic analysis of cellular fatty acid methyl esters appear to be more valuable tools for identification and classification of unknown aeromonads, and these methods have already proven to be useful in routine analyses $(5,25,26)$. Nevertheless, it has also been shown that the results obtained with these two chemotaxonomic techniques may not always be in perfect agreement with DNA-DNA hybridization data (5, 26). Moreover, since an essential part of current Aeromonas taxonomy is based on determining the levels of DNA relatedness within and between phenotypically defined groups (phe-

\footnotetext{
* Corresponding author. Mailing address: Laboratorium voor Microbiologie, Universiteit Gent, K.-L. Ledeganckstraat 35, B-9000 Ghent, Belgium. Phone: 329 2645121. Fax: 329 2645346. Electronic mail address: geert.huys@rug.ac.be.
}

nospecies), which has resulted in the description of at least 14 DNA hybridization groups (HGs), there is a tendency to look for additional genomic methods that are less laborious and require only small amounts of purified DNA.

Over the past 15 years, a significant number of DNA-based techniques have been introduced into the field of bacterial characterization and taxonomy. These genomic fingerprinting methods were developed to detect DNA sequence polymorphisms by using general principles, such as restriction endonuclease analysis, molecular hybridization, and PCR amplification. In a comparative study of different typing methods, Altwegg et al. (4) demonstrated that restriction fragment length polymorphism analysis and ribosomal DNA typing (ribotyping) were very successful in differentiating epidemiologically unrelated Aeromonas isolates. In a later paper Martinetti Lucchini and Altwegg (32) reported that ribotyping patterns can also be used as taxonomic markers and can be used to identify most Aeromonas strains at the HG level; unfortunately, in this study the ribotyping analysis relied on comparing band patterns by eye, which is not only time consuming but also often inaccurate. More recently, the randomly amplified polymorphic DNA technique was used to genetically differentiate Aeromonas strains that were isolated mainly from fish (35). Because only a limited number of species (Aeromonas hydrophila and Aeromonas salmonicida subsp. salmonicida) were included, additional fingerprinting data are needed to assess the usefulness of the randomly amplified polymorphic DNA technique for reliable identification of aeromonads.

Previously, we evaluated the use of the AFLP technique for identification and classification of bacteria at the subgeneric level (28). The concept of the AFLP technique was originally developed for plant-breeding purposes, but this technique is now considered a universal method for fingerprinting DNA from any source or DNA of any complexity, including human, animal, and plant DNAs $(44,47)$. Essentially, the AFLP method 
combines the principle of restriction fragment length polymorphism analysis with highly specific PCR amplification. Despite its obvious resemblance to restriction fragment length polymorphism, the name AFLP should in no way be considered an acronym because AFLP analysis in fact detects only the presence or absence of restriction fragments rather than length polymorphisms (44).

In this study, our aim was to investigate the taxonomic potential of the AFLP technique for discriminating the various HGs delineated in the genus Aeromonas. We therefore analyzed the AFLP patterns of 98 Aeromonas strains, representing all currently described genomic species in this genus, as well as the phenospecies Aeromonas allosaccharophila, Aeromonas encheleia, Aeromonas enteropelogenes, and Aeromonas ichthiosmia, which have not yet been allocated to particular HGs.

\section{MATERIALS AND METHODS}

Strains and culture conditions. All of the bacterial cultures used in this study are listed in Table 1. The strains used were either obtained from the Culture Collection of the Laboratorium voor Microbiologie, Universiteit Gent, Ghent, Belgium, or were kind gifts from M. Altwegg (Institute of Medical Microbiology, University of Zürich, Zürich, Switzerland), E. Falsen (Culture Collection of the University of Göteborg, Göteborg, Sweden), or B. Austin (Department of Biological Sciences, Heriot-Watt University, Riccarton, Edinburgh, Scotland). Most Aeromonas strains were grown aerobically on Trypticase soy agar containing $3 \%$ (wt/vol) Trypticase soy broth (BBL) and $1.5 \%$ (wt/vol) bacteriological agar no. 1 (Oxoid) overnight at $28^{\circ} \mathrm{C}$; the only exception was the psychrophilic species $A$. salmonicida, which was incubated at $22^{\circ} \mathrm{C}$. Plesiomonas shigelloides strains were grown at $37^{\circ} \mathrm{C}$.

Isolation and purification of DNA. Approximately $25 \mathrm{mg}$ (wet weight) of cells grown on Trypticase soy agar was washed with $500 \mu$ l of resuspension solution $(150 \mathrm{mM} \mathrm{NaCl}, 10 \mathrm{mM}$ EDTA; $\mathrm{pH} 8.0)$. The resulting cell suspension was centrifuged for 2 min at $14,926 \times g$ with a Heraeus Biofuge 13 centrifuge and resuspended in $100 \mu \mathrm{l}$ of TE buffer ( $10 \mathrm{mM}$ Tris-HCl, $1 \mathrm{mM}$ EDTA; $\mathrm{pH} 7.6$ ). The cells were lysed with $500 \mu \mathrm{l}$ of GES reagent $(5 \mathrm{M}$ guanidium thiocyanate$100 \mathrm{mM}$ EDTA $-0.5 \%$ [vol/vol] sarcosyl), and the DNA was extracted with chloroform-isoamyl alcohol $(24: 1, \mathrm{vol} / \mathrm{vol})$ essentially as described by Pitcher et al. (37). Total genomic DNA was precipitated with 0.54 volume of isopropanol, washed twice with $150 \mu \mathrm{l}$ of $70 \%$ (vol/vol) ethanol, and vacuum dried. Finally, DNA pellets were redissolved in $100 \mu \mathrm{l}$ of TE buffer and treated with $25 \mu \mathrm{l}$ of DNase-free RNase $\left(0.25 \mathrm{mg} \mathrm{ml}^{-1}\right)$ for $1.5 \mathrm{~h}$ at $37^{\circ} \mathrm{C}$. The DNA concentrations were determined by measuring the $A_{260}$ with a Uvikon model 940 spectrophotometer. The integrities and molecular weights of the DNA preparations were checked on a $1 \%$ agarose gel containing ethidium bromide $\left(0.5 \mu \mathrm{g} \mathrm{m}^{-1}\right)$ by using TAE (40 mM Tris-acetate, $1 \mathrm{mM}$ EDTA; $\mathrm{pH} 8.0$ ) as the electrophoresis buffer.

Preparation of template DNA for PCR. All protocols relating to the preparation of AFLP templates were performed essentially as described previously by Janssen et al. (28). Briefly, purified intact chromosomal DNA $(1 \mu \mathrm{g})$ was digested with ApaI and TaqI (Pharmacia Biotech, Uppsala, Sweden). Subsequently, double-stranded restriction-halfsite-specific adaptors were ligated to the restriction fragments by using T4 ligase (Pharmacia Biotech, Uppsala, Sweden). ApaI and Taq I adaptors were prepared by mixing equimolar amounts of the partially complementary oligonucleotide sequences 5'-TCGTAGACTGCGTACAGG CC-3' and 5'-TGTACGCAGTCTAC-3' (for ApaI) and 5'-GACGATGAGTCCTGAC-3' and 5'-CGGTCAGGACTCAT-3' (for TaqI). Completed ApaI-TaqI templates were precipitated with $1.25 \mathrm{M}$ ammonium acetate and $50 \%$ (vol/vol), isopropanol and washed with $70 \%$ ethanol before they were resuspended in 100 $\mu l$ of T0.1E buffer ( $10 \mathrm{mM}$ Tris, $0.1 \mathrm{mM}$ EDTA; pH 8.0).

AFLP reactions. ApaI-TaqI restriction fragments tagged with specific adaptors were used as template DNAs for selective PCR amplification directed by primers A01 (5'-GACTGCGTACAGGCCCA-3') and T01 (5'-CGATGAGTCCTGAC CGAA- $3^{\prime}$ ) (selective bases at the $3^{\prime}$ ends of the primers are underlined). The primer notations and amplification procedures used have been described previously (28). Primer A01 was labelled at its $5^{\prime}$ end with a T4 kinase (Pharmacia Biotech) assay by using [ ${ }^{32} \mathrm{P} \mid \mathrm{ATP}$ (Amersham International, Buckinghamshire, England) as described by Zabeau and Vos (47).

Electrophoresis and visualization of PCR products. Selectively amplified fragments were separated electrophoretically in a denaturing $5 \%$ polyacrylamide -8.3 $\mathrm{M}$ urea matrix (SequaGel; National Diagnostics) by using TBE (100 mM Tris, $100 \mathrm{mM}$ boric acid, 2 mM EDTA; $\mathrm{pH} 8.3$ ) as the electrophoresis buffer (28). The gels were vacuum dried with a gel dryer (model 583 ; Bio-Rad) for $55 \mathrm{~min}$ at $80^{\circ} \mathrm{C}$. The AFLP fingerprints were visualized autoradiographically by exposing the dried gels to Hyperfilm-MP (Amersham International) for 5 to $24 \mathrm{~h}$ depending on the amount of radiation determined with a regular Geiger-Müller counter. Following exposure, the film was developed with a model RGII X-ray film processor (Fuji, Tokyo, Japan).
Densitometric scanning and data processing. Autoradiograms were scanned by using a high-resolution densitoscanner (model RayVen RSU1; X-Ray Scanner Corp.). Transmission image data were stored in TIFF files and were further processed by using the GelCompar software, version 3.1 (Applied Maths, Kortrijk, Belgium). Following conversion, normalization, and background subtraction by using mathematical algorithms, levels of similarity between AFLP fingerprints were calculated by using the Pearson product-moment correlation coefficient applied to all points falling in the active zones of the band patterns. A cluster analysis was performed by using the unweighted pair group method with arithmetic averages. The GelCompar library manager was used to generate an AFLP database from representative band patterns.

\section{RESULTS AND DISCUSSION}

Complexity of AFLP patterns. ApaI-TaqI-digested genomic DNAs from 102 strains were used as templates for selective PCR amplification with primers A01 and T01, both of which have an adenine as a selective base at the $3^{\prime}$ end (see Materials and Methods). Our choice of restriction enzymes and primer sequences was based on the results of preliminary research $(24$, 28 ), from which we concluded that this specific endonucleaseprimer combination resulted in the visualization of about 40 to 60 PCR products per lane for Aeromonas genomes. We found that within this range, bands were evenly distributed along the length of the gel lane. This is important, since we have found that a good band distribution is critical for optimal normalization and a high level of discrimination in cluster analyses (28). In addition, whereas most of the ribotyping patterns obtained with Aeromonas strains (32) had only 10 to 15 bands per lane, the AFLP patterns obtained in this study are far more complex. Clearly, it is conceivable that a larger number of DNA polymorphisms favors more accurate comparative analysis results and thus strongly facilitates strain-to-strain discrimination.

The AFLP patterns of $P$. shigelloides, a taxon which has historically been closely associated with members of the genus Aeromonas (14), contained only 15 to 25 bands (data not shown). The explanation for this low number of bands lies in the fact that Plesiomonas genomes, which have a mean $\mathrm{G}+\mathrm{C}$ content of $51 \mathrm{~mol} \%$, are not as $\mathrm{G}+\mathrm{C}$ rich as the average Aeromonas genome (G+C content, 57 to $63 \mathrm{~mol} \%$ ) (14). Since the $\mathrm{G}+\mathrm{C}$ content of the ApaI recognition sequence is very high (GGGCC/C), it is predictable that $\mathrm{G}+\mathrm{C}$-rich genomes like the Aeromonas genomes will exhibit higher restriction rates and thus yield more bands under the PCR conditions used. Conversely, it has been shown that the frequency of TaqI cleavage ( $\mathrm{T} / \mathrm{CGA}$ ) is not significantly affected in the $\mathrm{G}+\mathrm{C}$ content range from 40 of $70 \mathrm{~mol} \%$ (28).

Reproducibility of AFLP patterns. It has been reported previously (28) that AFLP fingerprinting of bacterial genomes can be considered a highly reproducible technique that results in the generation of new databases for identification purposes. In this study, the level of reproducibility was determined by using the AFLP pattern of strain LMG 13459 as an internal reference during each electrophoresis run. Following normalization and subtraction of background values, we found that the intragel-specific correlation levels for the reference samples were between 95.0 and $98.5 \%$. Minimal differences in the correlation values between reference lanes were usually introduced by inevitable variations in background intensities and electrophoresis times. On the whole, these empirical parameters did not significantly affect the results derived from the cluster analysis.

Cluster analysis of AFLP data. Using the unweighted pair group method with arithmetic averages for clustering, we identified a total of 13 AFLP clusters (clusters I through XIII) when taxa were delineated at a correlation level of $43 \%$ (Fig. 1). Each of the clusters except clusters II, III, VIII, and IX, 
TABLE 1. Strains used

\begin{tabular}{|c|c|c|c|c|}
\hline Taxon & $\mathrm{HG}^{a}$ & Strain $^{b}$ & $\begin{array}{l}\text { Designation as received } \\
(\text { other designation }[\mathrm{s}])^{b}\end{array}$ & Source of isolation \\
\hline \multirow[t]{7}{*}{ A. hydrophila } & HG1 & LMG $2844^{\mathrm{T}}$ & $\operatorname{ATCC} 7966^{\mathrm{T}}\left({\left.\text { A } 306^{\mathrm{T}}\right)}\right.$ & Milk with fishy odor \\
\hline & & LMG 13439 & A 17 & Unknown \\
\hline & & LMG 13442 & A 52 & Feces \\
\hline & & LMG 13443 & A 229 & Feces \\
\hline & & LMG 13656 & A 82 & Feces \\
\hline & & LMG 13658 & A 162 & Feces \\
\hline & & LMG 13660 & A 188 & Bactec vial \\
\hline \multirow[t]{5}{*}{ A. hydrophila } & HG2 & LMG $13444^{c}$ & A 307 & Diseased fish \\
\hline & & LMG 13446 & A 13 & Environmental \\
\hline & & LMG 13447 & A 39 & Water \\
\hline & & LMG 13448 & A 169 & Feces \\
\hline & & LMG 13662 & A 295 & Feces \\
\hline \multirow[t]{6}{*}{ A. hydrophila } & HG3 & LMG 13449 & A 99 & Feces \\
\hline & & LMG 13450 & A 63 & Feces \\
\hline & & LMG $13451^{c}$ & A 308 & Freshwater \\
\hline & & LMG 13453 & A 8 & Environmental \\
\hline & & LMG 13674 & A 14 & Environmental \\
\hline & & LMG 13675 & A 15 & Environmental \\
\hline A. salmonicida subsp. salmonicida & HG3 & LMG $3780^{\mathrm{T}}$ & NCMB $1102^{\mathrm{T}}\left(\right.$ ATCC $\left.33658^{\mathrm{T}}\right)$ & Atlantic salmon \\
\hline A. salmonicida subsp. masoucida & HG3 & LMG $3782^{\mathrm{T}}$ & ATCC $27013^{\mathrm{T}}\left(\mathrm{NCMB} 2020^{\mathrm{T}}\right)$ & Oncorhynchus masou \\
\hline A. salmonicida subsp. achromogenes & HG3 & LMG $14900^{\mathrm{T}}$ & ATCC $33659^{\mathrm{T}}\left(\mathrm{NCMB} 1110^{\mathrm{T}}\right)$ & Brown trout \\
\hline A. salmonicida subsp. smithia & HG3 & LMG $16264^{\mathrm{T}}$ & CCM $4103^{\mathrm{T}}\left(\mathrm{ATCC} 49393^{\mathrm{T}}\right)$ & Ulcer on Rutilis rutilis \\
\hline \multirow[t]{7}{*}{ A. caviae } & HG4 & LMG $3775^{\mathrm{T}}$ & $\operatorname{ATCC} 15468^{\mathrm{T}}\left(\mathrm{A} 309^{\mathrm{T}}\right)$ & Epizootic guinea pig \\
\hline & & LMG 13454 & A 1 & Human \\
\hline & & LMG 13456 & A 41 & Feces \\
\hline & & LMG 13457 & A 102 & Feces \\
\hline & & LMG 13676 & A 12 & Environmental \\
\hline & & LMG 13678 & A 26 & Blood culture \\
\hline & & LMG 13680 & A 61 & Feces \\
\hline \multirow[t]{6}{*}{ A. caviae } & HG5A & LMG $13459^{c}$ & A 310 & Fish \\
\hline & & LMG 13460 & A 6 & Environmental \\
\hline & & LMG 13461 & A 75 & Feces \\
\hline & & LMG 13681 & A 163 & Feces \\
\hline & & LMG 13683 & A 208 & Feces \\
\hline & & LMG 13684 & A 225 & Feces \\
\hline \multirow{3}{*}{ A. caviae } & HG5B & LMG 13465 & A 117 & Feces \\
\hline & & LMG 13467 & A 242 & Feces \\
\hline & & LMG 13468 & A 284 & Feces \\
\hline \multirow[t]{4}{*}{ A. media } & HG5B & LMG $9073^{\mathrm{T}}$ & $\operatorname{ATCC} 33907^{\mathrm{T}}\left(\mathrm{A} 912^{\mathrm{T}}\right)$ & Water \\
\hline & & LMG 14687 & BA 91 & Fish farm, England \\
\hline & & LMG 14688 & BA 92 & Fish farm, England \\
\hline & & LMG 14689 & BA 93 & Fish farm, England \\
\hline \multirow{8}{*}{ A. eucrenophila } & HG6 & LMG $3774^{\mathrm{T}}$ & $\operatorname{NCMB} 74^{\mathrm{T}}\left(\mathrm{A} 311^{\mathrm{T}}\right)$ & Freshwater fish \\
\hline & & LMG 13057 & CCUG 30341 (A 914) & Unknown \\
\hline & & LMG 13058 & CCUG 30342 (A 1650) & Carp \\
\hline & & LMG 13060 & CCUG 30344 (A 1652) & Water \\
\hline & & LMG 13061 & CCUG 30345 (A 1653) & Water \\
\hline & & LMG 13062 & CCUG 30346 (A 1654) & Groundwater \\
\hline & & LMG 13687 & CCUG 30343 (A 1651) & Water \\
\hline & & LMG 13691 & CCUG 30347 (A 1655) & Groundwater \\
\hline \multirow[t]{2}{*}{ A. sobria } & HG7 & LMG $3783^{\mathrm{T}}$ & $\operatorname{CIP} 74.33^{\mathrm{T}}\left(\mathrm{A} 312^{\mathrm{T}}\right)$ & Fish \\
\hline & & LMG 13469 & A 915 & Fish \\
\hline \multirow[t]{11}{*}{ A. veronii biogroup sobria } & HG8 & LMG 13067 & CCUG 30356 (A 10) & Environmental \\
\hline & & LMG 13068 & CCUG 30357 (A 64) & Feces \\
\hline & & LMG 13070 & CCUG 30359 (A 68) & Feces \\
\hline & & LMG 13071 & CCUG 30360 (A 132) & Feces \\
\hline & & LMG 13072 & CCUG 30361 (A 151) & Feces \\
\hline & & LMG 13073 & CCUG 30362 (A 155) & Feces \\
\hline & & LMG 13074 & CCUG 30363 (A 916) & Feces \\
\hline & & LMG 13693 & A 11 & Environmental \\
\hline & & LMG 13694 & A 27 & Unknown \\
\hline & & LMG 13695 & A 28 & Feces \\
\hline & & LMG 13700 & A 168 & Feces \\
\hline \multirow[t]{4}{*}{ A. jandaei } & HG9 & LMG $12221^{\mathrm{T}}$ & ATCC $49568^{\mathrm{T}}\left(\mathrm{A} 1642^{\mathrm{T}}\right)$ & Feces \\
\hline & & LMG 13064 & CCUG 30348 (A 179) & Feces \\
\hline & & LMG 13065 & CCUG 30350 (A 919) & Feces \\
\hline & & LMG 13066 & CCUG 30351 (A 950) & Unknown \\
\hline
\end{tabular}


TABLE $1-$ Continued

\begin{tabular}{|c|c|c|c|c|}
\hline Taxon & $\mathrm{HG}^{a}$ & Strain ${ }^{b}$ & 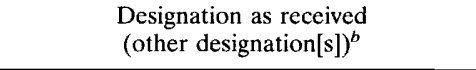 & Source of isolation \\
\hline & & LMG 13077 & CCUG 30352 (A 1641, ATCC 49570) & Wound below left eye (human) \\
\hline & & LMG 13079 & CCUG 30355 (A 1644, ATCC 49571) & Leg wound, diver \\
\hline \multirow[t]{5}{*}{ A. veronii biogroup veronii } & HG10 & LMG $9075^{\mathrm{T}}$ & $\operatorname{ATCC~} 35624^{\mathrm{T}}\left(\mathrm{A} 901^{\mathrm{T}}\right)$ & Near-drowning victim \\
\hline & & LMG 16183 & A 920 (CDC 1067-84) & Stool, New Mexico \\
\hline & & LMG 16332 & ATCC 35622 (CDC 0140-84) & Stool, Connecticut \\
\hline & & LMG 16333 & ATCC 35623 (CDC 0964-83) & Stool, Pennsylvania \\
\hline & & LMG 16334 & ATCC 35625 (CDC 1170-83) & Wound, Michigan \\
\hline \multirow[t]{2}{*}{ A. veronii-like } & HG11 & LMG 13075 & CCUG 30364 (A 902, ATCC 35941) & Ankle fracture \\
\hline & & LMG 13076 & CCUG 30365 (A 926) & Water, Mohawk River \\
\hline \multirow[t]{4}{*}{ A. schubertii } & HG12 & LMG $9074^{\mathrm{T}}$ & $\operatorname{ATCC} 43700^{\mathrm{T}}\left(\mathrm{A} 903^{\mathrm{T}}\right)$ & Abscess on forehead \\
\hline & & LMG 12655 & CCUG 25582 & Human leg wound \\
\hline & & LMG 12668 & CCUG 25583 & Human leg wound \\
\hline & & LMG 13473 & A 922 (ATCC 43701) & Human skin \\
\hline \multirow[t]{5}{*}{ A. trota } & HG13 & LMG $12223^{\mathrm{T}}$ & $\operatorname{ATCC} 49657^{\mathrm{T}}\left(\mathrm{A} 1646^{\mathrm{T}}\right)$ & Human stool, India \\
\hline & & LMG 13080 & CCUG 30366 (A 1645, ATCC 49659) & Appendix, California \\
\hline & & LMG 13081 & CCUG 30368 (A 1647, ATCC 49660) & Human stool, Thailand \\
\hline & & LMG 13082 & CCUG 30369 (A 1648, ATCC 49658) & Human stool, Bangladesh \\
\hline & & LMG 13083 & CCUG 30370 (A 1649, ATCC 49661) & Human stool, Indonesia \\
\hline A. enteropelogenes & $\mathrm{ND}^{d}$ & LMG $12646^{\mathrm{T}}$ & DSM $6394^{\mathrm{T}}$ & Human stool, India \\
\hline A. ichthiosmia & ND & LMG $12645^{\mathrm{T}}$ & DSM $6393^{\mathrm{T}}$ & Surface water \\
\hline \multirow[t]{3}{*}{ A. allosaccharophila } & ND & LMG 14021 & ATCC 35942 & Human stool, South Carolina \\
\hline & & LMG 14058 & CECT 4200 & Diseased elver, Valencia, Spain \\
\hline & & LMG $14059^{\mathbf{T}}$ & CECT $4199^{\mathrm{T}}$ & Diseased elver, Valencia, Spain \\
\hline \multirow[t]{4}{*}{ A. encheleia } & ND & LMG 16328 & CECT 4340 (S 177) & European eel, Valencia, Spain \\
\hline & & LMG 16329 & CECT 4341 (S 176) & European eel, Valencia, Spain \\
\hline & & LMG $16330^{\mathrm{T}}$ & CECT $4342^{\mathrm{T}}\left(\mathrm{S} 181^{\mathrm{T}}\right)$ & European eel, Valencia, Spain \\
\hline & & LMG 16331 & CECT 4343 (S 191) & European eel, Valencia, Spain \\
\hline \multirow[t]{4}{*}{ Aeromonas sp. } & ND & LMG 13470 & A 925 & Kidney of tarbot \\
\hline & & LMG 13471 & A 923 & Kidney of brook trout \\
\hline & & LMG 13472 & A 924 & Kidney of rainbow trout \\
\hline & & LMG 16184 & A 921 (CDC 0715-84) & Stool, South Carolina \\
\hline \multirow[t]{4}{*}{ P. shigelloides } & & LMG $4242^{\mathrm{T}}$ & NCTC $10360^{\mathrm{T}}\left(\right.$ ATCC $\left.14029^{\mathrm{T}}\right)$ & Dog feces \\
\hline & & LMG 12657 & CCUG 12881B & Human feces \\
\hline & & LMG 12658 & CCUG 12890 & Human cholecystitis \\
\hline & & LMG 12674 & CCUG 10616D & Aquarium sludge \\
\hline
\end{tabular}

\footnotetext{
${ }^{a} \mathrm{HG}$, as defined at the Centers for Disease Control (19).
}

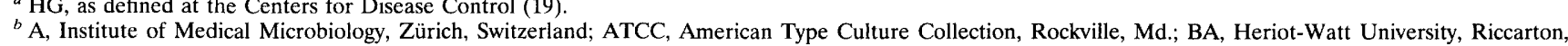
Edinburgh, Scotland; CCM, Czech Collection of Microorganisms, Masaryk University, Brno, Czech Republic; CCUG, Culture Collection of the University Göteborg, Göteborg, Sweden; CDC, Centers for Disease Control, United States Public Health Service, Atlanta, Ga.; CECT, Coleccion Espanola de Cultivos Tipo, Universidad de Valéncia, Valencia, Spain; CIP, Collection bactérienne de l'Institut Pasteur, Paris, France; DSM, Deutsche Sammlung von Mikroorganismen und Zellkulturen GmbH, Braunschweig, Germany; LMG, Culture Collection of the Laboratorium voor Microbiologie Gent, Universiteit Gent, Ghent, Belgium; NCMB, National Collection of Marine Bacteria, Aberdeen, Scotland; NCTC, National Collection of Type Cultures, Central Public Health Laboratory, London, United Kingdom; S, Departamento de Microbiologia y Ecologia, Universitat de Valéncia, Valencia, Spain.

${ }^{c} \mathrm{HG}$ reference strain.

${ }^{d} \mathrm{ND}$, not defined.

which are discussed below, contained strains belonging to one HG. Within these genotypically defined groups, each strain usually produced a unique band pattern which could be used as a genomic fingerprint for bacterial identification and typing at the strain level. In some cases, however, the AFLP patterns of strains belonging to an $\mathrm{HG}$ appeared to be very similar, if not identical. For instance, strains LMG 14688 and LMG 14689 (cluster V) grouped together at a correlation level of $98.1 \%$ on the basis of their very similar band patterns (Fig. 1). Since these two isolates originated from the same site (Table 1) and were probably isolated at the same time, it is possible that these strains represent the same bacterial clone. Clearly, additional AFLP-based research (e.g., research in which other primer combinations are used) can be performed to determine the true clonal identity of strains which produced very similar patterns in this study. Below, all AFLP clusters shown in Fig. 1 are discussed with regard to their taxonomic significance and their relationship to DNA-DNA hybridization results and other data.
Together, clusters I and II comprise eight strains belonging to Aeromonas eucrenophila (HG6), four A. encheleia strains, and two isolates allocated to genomic species 11 (Aeromonas veronii-like); the latter taxon was mentioned for the first time by Hickman-Brenner et al. (21) in the original description of the ornithine decarboxylase-positive $\left(\mathrm{ODC}^{+}\right)$species Aeromonas veronii (now $A$. veronii biogroup veronii HG10). HickmanBrenner et al. found that human $\mathrm{ODC}^{+}$isolate LMG 13075 does not belong to $A$. veronii because its level of relatedness to the type strain of HG10 (strain LMG 9075) was much less than $70 \%$ at $60^{\circ} \mathrm{C}$ as determined by DNA hybridization. Ever since, this strain has been placed in HG11 together with environmental isolate LMG 13076, and these strains may represent an unnamed species belonging to the genus Aeromonas. More recently, phenotypic (10) and chemotaxonomic (26) evidence has shown that HG11 does not belong to $A$. veronii biogroup veronii but belongs to the Aeromonas caviae complex (which comprises HG4, HG5A, HG5B, and HG6). Our AFLP data confirmed these findings because strains LMG 13075 and 


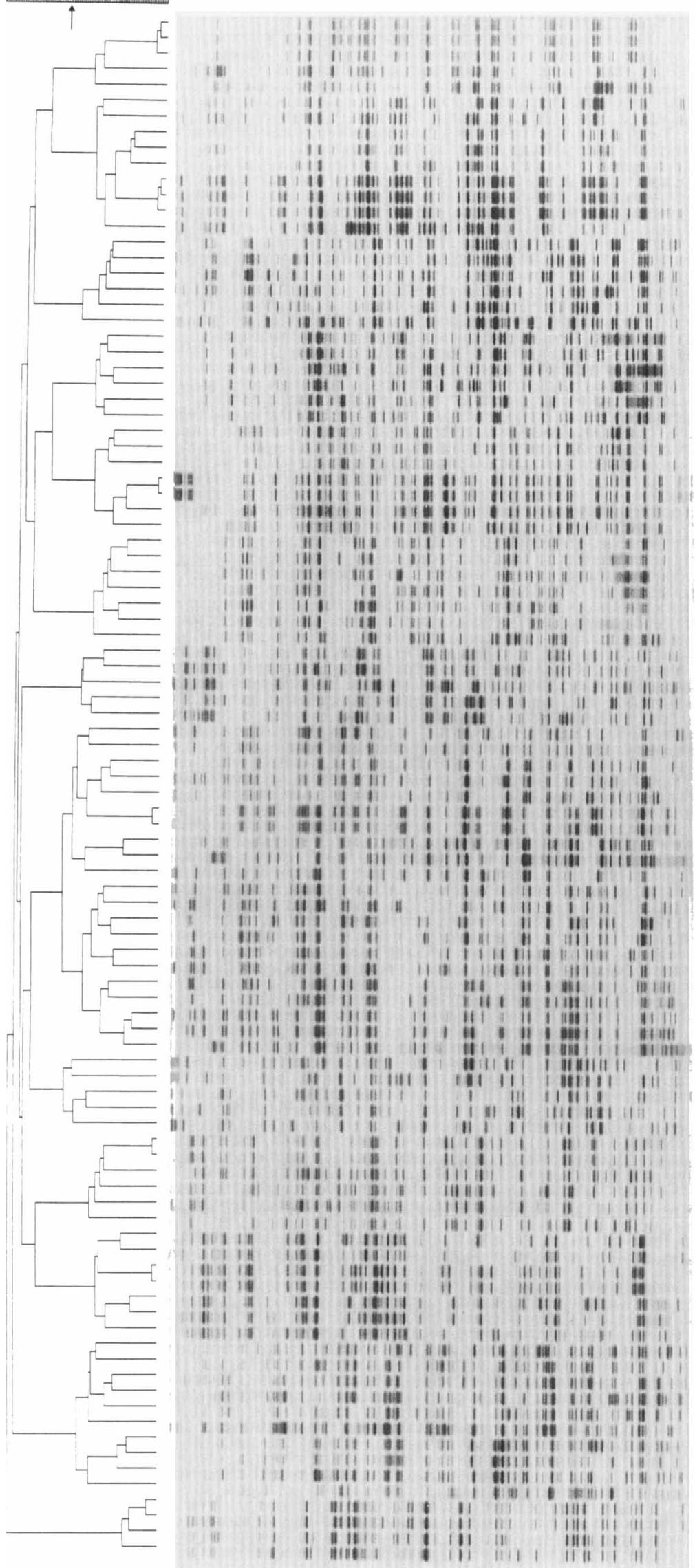

A. encrenophila HG6 LMG 13057

A. etcrenophila HG6 LMG 3774

A. actrenophina HG6 LMG 13058

A. eucrenophila HG6 LMG 1368

A. veronii HGII LMG 13075

A. veromii HGII LMG 1307

A. cucrenophila HG6 LMG 13061

A. écrenophila HG6 LMG 1369

A. e'ucrenephila HG6 LMG 13062

A. encheleta LMG 16330

A. encheleta LMG 16331

A. encheleia LMG 16328

A. trota HG13 LMG 12223

A. trota HGI3

A. trota $\mathrm{HG}_{3} 3 \mathrm{LMG} 13082$

A. enteropelosenes LMG $12066^{\prime}$

A. trow HG13 LMG 13083

A. caviae HG5A LMG 13683

A. caviae HGSA LMG 1345\%

A. caviae HG5A LMG 1368

A. caviae HGSA LMG 13684

A. caviae HG5A LMG 1346

A. caviae HGSA LMG 13460

A. caviae HG5B LMG 13465

A. caviae HG5B LMG 13467

A. caviae HG5B LMG 13468

A. media HG5B LMG 1468

A. media HG5B LMC 14687

A. media HG5B LMG 9073'

A. caviae HG4 LMG 13457

A. caviae HG4 LMG 13678

A. caviae HG4 LMG 13680

A. caviae HG4 LMG $3775^{\prime}$

A. caviae HG4 LMG 13454

A. caviae HG4 LMG 13676

A. hydrophila HG2 LMG 13446

A. hydrophila HG2 LMG 1344

A. hydrophila HG2 LMG 13662

A. hydrophila HG2 LMG $13444^{*}$

A. hydrophila HG2 LMG 13447

A. veronii biogroup sobria HG8 LMG 1307|

A. veronii biogroup sobria HG8 LMG 13072

A. veronii biogroup sobria HG8 LMG 13073

A. veronii biogroup sobria HG8 LMG 13700

A. veronii biogroup sobria HG8 LMG 13070

A. allosaccharophila LMG 14058

A. allosaccharophila LMG 14059

A. sp. LMG 16184

A.

A. veronii biogroup sobria HG8 LMG 13074

A. veronii biogroup sobria HG8 LMG 13068

A. veronii biogroup sobria HG8 LMG 13693

A. veronii biogroup sobria $\mathrm{HC8}$

A. teronii biogroup sobria HG8 LMG 13694

A. veronii biogroup sobria HG8 LMG 13067

A. eronii biogroup veronii HG10 LMG 16334

VIIIb

A. veronii biogroup veroni HG10 LMG 1633

A. veronii biogroup veronii HGI0 LMG 16332

A. veromic bigroup veronii HGI0 LMG 9075

A. veronii biogroup veronii $\mathrm{HG} 10$ LMG 16183

A. sobria HG7 LMG 13469

A. sp. LMG 13470

A. sp. LMG 1347

A. sp. LMG 13472

A. jandaei HG9 LMG 12221

A. jandaei HG9 LMG 13064

A. jandaei HG9 LMG 13065

A. jandaci HG9 1 MG 13079

A. jandaei HG9 LMG 13077

A. hydrophila HGI LMG 13660

A. hvdrophila HGI LMG 13658

A hedrophila HGI LMG 2844

A. hvdrophila HGI LMG 13439

A. Marophila HGI LMG 13443

A. hydrophila HGI LMG 1344 2

A. hydrophila HG3 LMG 13449

A. Madrophita HG3 LMG 1345 1

A. hodrophila HG $\mathrm{HG}$ L.MG 13674

A. Mvarophata HG.3 LMG 1367

A. hadrophila HG.3 L.MGi 13453

A. hadropihila $\mathrm{HG}_{3}$ LMG 13450

I

II

III

IV

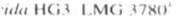

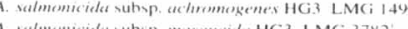

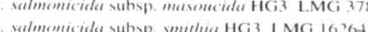

A. schubertii HG 12 LMG 126008

A whe stii HG12 LMG 1347 .

A. whtubernii HGi12 LMG 12055

VIIIa

VII

$\mathbf{X}$ 
LMG 13076 were found in cluster II and clearly grouped together with three representatives of HG6. In addition, cluster II also contains the four isolates recovered from European eels (strains LMG 16328, LMG 16329, LMG $16330^{\mathrm{T}}$ [T = type strain], and LMG 16331) which were recently found to belong to a new Aeromonas species named $A$. encheleia (13). We found that in the AFLP analysis A. encheleia LMG 16329, LMG $16330^{\mathrm{T}}$, and LMG 16331 produced very similar band patterns which had a correlation level of $95.3 \%$, as determined by cluster analysis. Strain LMG 16328 , whose level of DNA homology with labeled LMG $16330^{\mathrm{T}}$ DNA was only $70 \%$ (13), differed somewhat from strains LMG $16329, \mathrm{LMG} 16330^{\mathrm{T}}$, and LMG 16331, as shown in Fig. 1. However, all four A. encheleia strains were clearly members of the same AFLP cluster (cluster II), which also includes members of HG6 and HG11. Thus, the results described above are not consistent with the genotypic and phenotypic data from which Esteve et al. (13) concluded that strains LMG 16328, LMG 16329, LMG $16330^{\mathrm{T}}$, and LMG 16331 belong to a separate Aeromonas species. To further examine the observed discordance between DNA homology results and AFLP data, it would be worthwhile to perform additional DNA-DNA hybridization experiments with $A$. encheleia and A. eucrenophila in which well-characterized HG6 reference strains other than type strain LMG 3774 are used (13). In fact, our AFLP data indicated that LMG $3774^{\mathrm{T}}$ was a member of cluster I, which also includes four other $A$. eucrenophila strains. The level of correlation for clusters I and II is only $35.2 \%$, which is about $8 \%$ below the delineation level, as shown in Fig. 1. From the results described above, it could be suggested that the phenospecies $A$. eucrenophila actually consists of two subgroups which, at the moment, can be separated only genotypically by AFLP fingerprinting. A polyphasic taxonomic study is under way to further examine the possible subdivision of HG6, as well as to clarify the taxonomic positions of genomic species 11 and $A$. encheleia in the genus Aeromonas.

Cluster III contains five strains belonging to the ampicillinsusceptible (Amps) species Aeromonas trota (HG13) and the type strain of $A$. enteropelogenes (LMG 12646). The latter species was originally proposed by Schubert et al. (41) for a group of Aeromonas sobria-like psychrotrophic aeromonads which can be phenotypically and genotypically separated from all other Aeromonas species that have been described. However, Schubert et al. provided DNA-DNA homology data for only three other species ( $A$. hydrophila, $A$. sobria, and $A$. veronii) and thus did not include all members of the genus Aeromonas in their study. Moreover, phylogenetic evidence (11) has brought into serious question the species status of $A$. enteropelogenes by revealing that the $16 \mathrm{~S}$ rRNA sequence of LMG $12646^{\mathrm{T}}$ exhibits $100 \%$ similarity with the $16 \mathrm{~S}$ rRNA sequence of the type strain of $A$. trota (strain LMG 12223). Consequently, Collins et al. (11) suggested that additional DNADNA hybridization studies should be performed in order to determine the true genomic relatedness of the two taxa. To some extent, we addressed this problem by placing the type strain of $A$. enteropelogenes in cluster III on the basis of its AFLP pattern. In addition, Carnahan (8) mentioned that the phenotypic profile of $A$. enteropelogenes (41) is consistent with the biochemical properties of $A$. trota (9). However, no data concerning the susceptibility of $A$. enteropelogenes strains to ampicillin have been published.

Clusters IV and V contain HG5A (phenotypically $A$. caviae) and HG5B (phenotypically Aeromonas media or A. caviae), respectively; the correlation level for these taxa is $35.2 \%$. This relatively high level of linkage reflects the fact that these two groups are more closely related as determined by DNA-DNA hybridization than any other Aeromonas HGs (3). In fact, it has been suggested (6) that HG5A and HG5B should be considered two subspecies of $A$. media, since HG5B contains the type strain of $A$. media (strain LMG 9073). To examine this proposal, the AFLP technique should prove to be a valuable tool for discriminating HG5A and HG5B at a high level of resolution. In addition, we determined that the striking biochemical and physiological differences between the nonmotile $A$. media strains (which do not utilize sucrose as a sole carbon source and produce a brown diffusible pigment) and the motile $A$. caviae isolates obtained from clinical sources (which utilize sucrose as a sole carbon source and do not produce a brown diffusible pigment) belonging to HG5B are consistent with the fact that all four $A$. media strains included in our study (Table 1) belong to a separate subgroup within cluster V (Fig. 1). Moreover, these results may indicate that HG5B should be subdivided into two biogroups (6), analogous to the taxonomic position of $A$. veronii in cluster VIII (see below).

Cluster VI comprises seven strains, mainly from clinical sources, which have been classified as members of $A$. caviae HG4. As described above, members of the phenospecies $A$. caviae exhibit a wide range of genomic relatedness values and, as a result, have been placed in HG4, HG5A, and HG5B. However, the type strain of $A$. caviae (strain LMG 3775), is a member of HG4 and can also be differentiated from HG5 by phenotypic (29) and chemotaxonomic (26) characteristics.

Clusters VII, XI, and XII are composed of strains belonging to $\mathrm{HG} 2, \mathrm{HG} 1$, and $\mathrm{HG} 3$, respectively, of the $A$. hydrophila complex. Ever since Popoff et al. (38) delineated three DNA homology groups (now HG1, HG2, and HG3) in the phenospecies $A$. hydrophila, workers have questioned whether these groups can also be differentiated by phenotypic characteristics. Over the years, several studies $(1,6,15)$ have indeed shown that a limited set of biochemical tests can be used to distinguish HG1, HG2, and HG3. As a result, strains belonging to HG1 (cluster XI) have been placed in the type species of the genus Aeromonas, $A$. hydrophila, since HG1 includes the type strain of this phenospecies (strain LMG 2844). Along with the DNADNA hybridization results (38), the currently available phenotypic data indicate that $\mathrm{HG} 2$ (cluster VII) also deserves recognition as a separate phenospecies of the genus Aeromonas rather than recognition as an unnamed genomic subgroup of A. hydrophila. In fact, Ali et al. (2) proposed the name "Aeromonas bestiarum" at the Fourth International Symposium on Aeromonas and Plesiomonas; the specific epithet of this taxon refers to the fact that most $\mathrm{HG} 2$ strains originate from environmental and veterinary sources. Moreover, these authors suggested that a cutoff DNA-DNA homology value of $85 \%$ at $60^{\circ} \mathrm{C}$ allowed them to separate " $A$. bestiarum" from all other Aeromonas species. In close agreement with these findings, Hänninen (15) reported that the levels of DNA relatedness between a collection of mainly environmental HG2 strains and

FIG. 1. Dendrogram derived from an unweighted average pair group cluster analysis of the AFLP patterns of all 98 Aeromonas strains included in this study. The tracks show the processed band patterns after conversion, normalization, and subtraction of background. Levels of linkage are expressed as the Pearson productmoment similarity coefficients. The level of cluster delineation (43\%) is indicated by an arrow. As indicated in the text, HG11 is phenotypically similar to A. veronii, but represents an unnamed Aeromonas species. 
the reference strain of $A$. hydrophila HG3 (strain LMG 13451) were 45 to $87 \%$ at the optimal temperature $\left(60^{\circ} \mathrm{C}\right)$. Clearly, these data suggest that $\mathrm{HG} 2$ is genotypically more closely related to HG1 and HG3 than Popoff et al. (38) realized. Moreover, since the DNA homology values for members of the HGs belonging to the $A$. hydrophila complex $(2,15)$ do not strictly support the phylogenetic definition of a bacterial species (46), perhaps subspecies rank should be considered for HG2. However, the AFLP results in Fig. 1 leave no doubt about the genotypic separation of the HG2 strains in cluster VII from HG1 and HG3, which belong in clusters XI and XII, respectively. On the other hand, the taxonomic position of HG3 is complicated by the fact that two easily separable Aeromonas phenospecies apparently exhibit enough DNA relatedness to be classified in the same DNA homology group. It could be suggested that HG3 should correspond to the name $A$. salmonicida because this HG contains the type strains of $A$. salmonicida subsp. salmonicida (LMG 3780), $A$. salmonicida subsp. achromogenes (LMG 14900), A. salmonicida subsp. masoucida (LMG 3782), and $A$. salmonicida subsp. smithia (LMG 16264), which represent a group of psychrophilic, nonmotile, fish-associated aeromonads. Altwegg et al. (6) suggested that $A$. hydrophila HG3 should be classified in a new subspecies of $A$. salmonicida, although it has never been reported that motile HG3 strains are strictly fish associated or might represent a potential danger to fish farm industries. Still, it is clear that each proposal for the naming of HG3 should take into account both the phenotypic diversity and the genetic similarity of the constituent subgroups without introducing any confusion by nomenclatural changes.

Cluster VIII is defined by the AFLP patterns of 21 strains that belong to the phenospecies $A$. veronii biogroup sobria (HG8), A. veronii biogroup veronii (HG10), A. ichthiosmia, and $A$. allosaccharophila. It has been reported previously (6) that $A$. veronii biogroup sobria and $A$. veronii biogroup veronii exhibit a very high level of genomic relatedness, which results in the placement of both taxa in the same hybridization group (HG8/10). On the other hand, the striking phenotypic diversity within $\mathrm{HG} 8 / 10$ has led to the conclusion that $A$. veronii should be split into two biogroups corresponding to HG8 and HG10. Consistent with the DNA-DNA hybridization results, the AFLP patterns of cluster VIII strains (Fig. 1) do not permit clear-cut differentiation between the two biogroups. A similar phenomenon has been reported by Altwegg et al. $(5,6)$, who noticed that many of the HG8/10 strains used in our study were actually spread over several phena when they performed a cluster analysis with data derived from multilocus enzyme electrophoresis. In fact, we determined that phena 3 and 4 revealed by multilocus enzyme electrophoresis $(5,6)$ correspond to some extent to clusters VIIIa and VIIIb, respectively (Fig. 1). For instance, cluster VIIIa strains LMG 13073, LMG 13074, and LMG 16184 were also members of phenon 3. However, strain LMG 16184 has never been unequivocally assigned to HG8/10 because it exhibited only $69 \%$ DNA relatedness with the type strain of HG10 (strain LMG 9075) under optimal reassociation conditions (21). Therefore, it has been suggested that strain LMG 16184 has diverged from strain LMG $9075^{\mathrm{T}}$, which belongs to cluster VIIIb. This latter cluster also includes the type strain of $A$. ichthiosmia (strain LMG 12645), a psychrotrophic species that consists of $A$. sobria-like aeromonads (42). Like the species rank of $A$. enteropelogenes (see above), the species rank of $A$. ichthiosmia is still open to question because the $16 \mathrm{~S}$ rRNA gene sequence of $\mathrm{LMG} 12645^{\mathrm{T}}$ is identical to that of the type strain of $A$. veronii (11). Our fingerprinting data again support the phylogenetic results, despite the inability of the AFLP technique to distinguish be- tween the two $A$. veronii biogroups. Since all strains designated $A$. ichthiosmia lack the potential for ornithine decarboxylation and esculin hydrolysis (42), two key tests which are uniformly positive for $A$. veronii biogroup veronii isolates (21), it seems reasonable to classify $A$. ichthiosmia in $A$. veronii biogroup sobria on the basis of phenotypic characteristics. Still, additional DNA homology studies are needed to verify this classification. Likewise, DNA-DNA hybridizations have also been used in an attempt to clarify the taxonomic position of $A$. allosaccharophila, a recently described mesophilic member of the genus Aeromonas (12) which belongs to cluster VIIIa. In the original description of this species (33), Martinez-Murcia et al. concluded on the basis of phenotypic and phylogenetic data that three biochemically atypical Aeromonas strains (LMG 14021, LMG 14058, and LMG $14059^{\mathrm{T}}$ ), which have identical 16S rRNA sequences, in fact represent a new genomic species closely related to $A$. sobria and $A$. veronii. On the basis of DNA homology data (12), A. allosaccharophila was found to be a very homogeneous taxon, although unlabelled DNA from strain LMG 14021 exhibited only $70 \%$ homology under optimal reassociation conditions with labelled DNA from the type strain (strain LMG 14059). Strains LMG 14058 and $\mathrm{LMG} 14059^{\mathrm{T}}$, on the other hand, exhibited a level of relative genomic relatedness of $100 \%$. In close agreement with these findings, the latter two strains also produced very similar AFLP patterns which were tightly linked at a correlation level of $96.2 \%$ (Fig. 1). Strain LMG 14021, however, was more closely related to $A$. veronii LMG 13074 and LMG 16184. Solely on the basis of AFLP data, it appears that $A$. allosaccharophila does not represent an additional genotypic group in the genus Aeromonas, as would be expected from DNA-DNA hybridization data (12), but rather should be considered a new phenospecies which seems genetically similar to HG8 and HG10. In light of these findings, it is our opinion that more DNA homology data are required to justify definition of $A$. allosaccharophila as HG14 (12).

Cluster IX joins two representatives of $A$. sobria HG7 with Aeromonas sp. strains LMG 13470, LMG 13471, and LMG 13472 at a correlation level of $45 \%$. According to the phenotypic data of Kämpfer and Altwegg (29), strains LMG 13470, LMG 13471, and LMG 13472 are closely related to the type strain of $A$. veronii biogroup veronii HG10 (strain LMG 9075) and constitute a separate phenon in the genus Aeromonas. Since all of the strains in this phenon were biochemically distinct from HG8, Kämpfer and Altwegg considered them representatives of HG10. Consequently, strains LMG 13470 , LMG 13471, and LMG 13472 were listed as members of $A$. veronii HG10 in our recent chemotaxonomic study of the use of fatty acid methyl ester profiles for discriminating between phenospecies and HGs in the genus Aeromonas (26); together with LMG $9075^{\mathrm{T}}$, these three strains were recovered in a separate fatty acid methyl ester cluster which allowed clear differentiation from HG8 isolates. However, it should be noted that this cluster was obtained by using mean fatty acid methyl ester data instead of individual fatty acid profiles. In fact, a cluster analysis of the individual fatty acid methyl ester profiles revealed that the type strain of HG10 (strain LMG 9075) is in no way similar to strains LMG 13470, LMG 13471, and LMG 13472 (23). This finding was readily confirmed by the results of our AFLP study (Fig. 1). In addition, it has been reported previously (5) that the five strains in AFLP cluster IX (Fig. 1) also constitute a distinct phenon in the genus Aeromonas on the basis of the results of a multilocus enzyme electrophoresis analysis. From both the genotypic point of view and the chemotaxonomic point of view it thus appears that strains LMG 13470, LMG 13471, and LMG 13472 may represent a new and 
currently undefined Aeromonas taxon which is phenotypically similar to HG10 (29) but seems genotypically more closely related to HG7. Notwithstanding this, proper taxonomic allocation of these strains in the genus Aeromonas will require intensive DNA homology studies and additional phenotypic research.

Clusters $X$ and XIII comprise representative strains of the phenospecies Aeromonas jandaei (HG9) and Aeromonas schubertii (HG12), respectively. The majority of these strains originated from human specimens (including feces, blood, skin, and wounds [Table 1]), illustrating the clinical significance of HG9 and HG12. In the original descriptions of $A$. jandaei (7) and $A$. schubertii (20), it was stated that both of these taxa have unique biochemical profiles which provide a limited number of simple tests for differentiation from other Aeromonas sp. On genotypic grounds, HG9 and HG12 indeed constitute two homogeneous AFLP clusters (Fig. 1), which confirms their taxonomic standing in the genus Aeromonas.

In conclusion, in this investigation we clearly elucidated the taxonomic significance and discriminative power of the AFLP technique for differentiating the current members of the genus Aeromonas. Moreover, the high overall level of correlation between our fingerprinting results and the previously published DNA hybridization data indicates that the AFLP concept can be considered a relatively fast, reproducible, high-resolution alternative to conventional DNA-DNA hybridization at the subgeneric level. In addition, some of the AFLP data presented in this paper strongly suggest that this technique will prove to be a valuable tool for refining the existing Aeromonas taxonomy. Most of the AFLP patterns produced in this study were used as representative library entries to generate a new HG-specific genotypic database named AERO94. At this time, we are evaluating the usefulness of the AERO94 library for identifying unknown Aeromonas isolates that originate from a wide range of environmental and clinical habitats.

\section{ACKNOWLEDGMENTS}

This research was carried out in the framework of contract G.O.A. 91/96-2 with the Ministerie van de Vlaamse Gemeenschap, Bestuur Wetenschappelijk Onderzoek (Belgium). G.H. acknowledges the support received from the Vlaams Instituut voor de bevordering van Wetenschaelijk-Technologisch onderzoek in de industric (Belgium) in the form of a bursary for advanced study. P.J. is indebted to the European Environmental Research Organisation (The Netherlands) for a short-term fellowship.

We also thank L. Vauterin and D. Dewettinck for technical assistance in the preparation of Fig. 1.

\section{REFERENCES}

1. Abbott, S. L., W. K. W. Cheung, S. Kroske-Bystrom, T. Malekzadeh, and M. J. Janda. 1992. Identification of Aeromonas strains to the genospecies level in the clinical laboratory. J. Clin. Microbiol. 30:1262-1266.

2. Ali, A., A. M. Carnahan, J. M. Janda, M. Altwegg, and S. W. Joseph. 1993. Proposal of a new Aeromonas sp., Aeromonas bestiantm sp. nov (formerly DNA hybridization group 2), abstr. P-1, p. 35. In Fourth International Symposium on Aeromonas and Plesiomonas, 15 to 16 May 1993, Atlanta, Ga.

3. Altwegg, M. 1990. Taxonomy and epidemiology of Aeromonas spp.: the value of new typing methods. Habilitationsschrift, Universität Zürich, Zurich.

4. Altwegg, M., R. Altwegg-Bissig, A. Demarta, R. Peduzzi, M. W. Reeves, and B. Swaminathan. 1988. Comparison of four typing methods for Aeromonas species. J. Diarrhoeal Dis. Res. 6:88-94.

5. Altwegg, M., M. W. Reeves, R. Altwegg-Bissig, and D. J. Brenner. 1991 Multilocus enzyme analysis of the genus Aeromonas and its use for species identification. Zentralbl. Bakteriol. 275:28-45.

6. Altwegg, M., A. G. Steigerwalt, R. Altwegg-Bissig, J. Lüthy-Hottenstein, and D. J. Brenner. 1990. Biochemical identification of Aeromonas genospecies isolated from humans. J. Clin. Microbiol. 28:258-264.

7. Carnahan, A., G. R. Fanning, and S. W. Joseph. 1991. Aeromonas jandaei (formerly genospecies DNA group $9 \mathrm{~A}$. sobria), a new sucrose-negative species isolated from clinical specimens. J. Clin. Microbiol. 29:560-564.

8. Carnahan, A. M. 1993. Aeromonas taxonomy: a sea of change, abstr. 5, p. 13.
In Fourth International Symposium on Aeromonas and Plesiomonas, 15 to 16 May 1993, Atlanta, Ga.

9. Carnahan, A. M., T. Chakraborty, G. R. Fanning, D. Verma, A. Ali, J. M. Janda, and S. W. Joseph. 1991. Aeromonas trota sp. nov., an ampicillinsusceptible species isolated from clinical specimens. J. Clin. Microbiol. 29. 1206-1210.

10. Carnahan, A. M., and S. W. Joseph. 1993 . Systematic assessment of geographically and clinically diverse aeromonads. Syst. Appl. Microbiol. 16:72-84.

11. Collins, M. D., A. J. Martinez-Murcia, and J. Cai. 1993. Aeromonas enteropelogenes and Aeromonas ichthiosmia are identical to Aeromonas trota and Aeromonas veronii, respectively, as revealed by small-subunit rRNA sequence analysis. Int. J. Syst. Bacteriol. 43:855-856.

12. Esteve, C., M. C. Gutiérrez, and A. Ventosa. 1995. DNA relatedness among Aeromonas allosaccharophila strains and DNA hybridization groups of the genus Aeromonas. Int. J. Syst. Bacteriol. 45:390-391.

13. Esteve, C., M. C. Gutiérrez, and A. Ventosa. 1995. Aeromonas encheleia sp. nov., isolated from European eels. Int. J. Syst. Bacteriol. 45:462-466.

14. Farmer, J. J., III, M. J. Arduino, and F. W. Hickman-Brenner. 1992. The genera Aeromonas and Plesiomonas, p. 3012-3045. In A. Balows, H. G. Trüper, M. Dworkin, W. Harder, and K. H. Schleifer (ed.), The prokaryotes, vol. 3. Springer-Verlag, New York.

15. Hänninen, M.-L. 1994. Phenotypic characteristics of the three hybridization groups of Aeromonas hydrophila complex isolated from different sources. J. Appl. Bacteriol. 76:455-462.

16. Havelaar, A. H., F. M. Schets, A. van Silfhout, W. H. Janssen, G. Wieten, and D. van der Kooij. 1992. Typing of Aeromonas strains from patients with diarrhoea and from drinking water. J. Appl. Bacteriol. 72:435-444.

17. Havelaar, A. H., J. F. M. Versteegh, and M. During. 1990. The presence of Aeromonas in drinking water supplies in the Netherlands. Zentralbl. Bakteriol. Hyg. 190:236-256.

18. Hazen, T. C., M. L. Raker, G. W. Esch, and C. B. Fliermans. 1978. Ultrastructure of red-sore lesions on largemouth bass (Micropterus salmoides): the association of the peritrich Epistylis sp. and the bacterium Aeromonas hydrophila. J. Protozool. 25:351-355.

19. Hickman-Brenner, F. W., G. R. Fanning, M. J. Arduino, D. J. Brenner, and J. J. Farmer III. 1988. Aeromonas group 501, a new mannitol-negative species found in human clinical specimens, abstr. P-20, p. 51. In second Workshop on Aeromonas and Plesiomonas, 7, May 1988, Miami Beach, Fla.

20. Hickman-Brenner, F. W., G. R. Fanning, M. J. Arduino, D. J. Brenner, and J. J. Farmer III. 1988. Aeromonas schubertii, a new mannitol-negative species found in human clinical specimens. J. Clin. Microbiol. 26:1561-1564.

21. Hickman-Brenner, F. W., K. L. MacDonald, A. G. Steigerwalt, G. R. Fanning, D. J. Brenner, and J. J. Farmer III. 1987. Aeromonas veronii, a new ornithine decarboxylase-positive species that may cause diarrhea. J. Clin. Microbiol. 25:900-906.

22. Holmberg, S. D., W. L. Schell, G. R. Fanning, I. K. Wachsmuth, F. W. Hickman-Brenner, P. A. Blake, D. J. Brenner, and J. J. Farmer. 1986. Aeromonas intestinal infections in the United States. Ann. Intern. Med. 105:683689

23. Huys, G. Unpublished data.

24. Huys, G., R. Coopman, M. Vancanneyt, I. Kersters, W. Verstraete, K. Kersters, and P. Janssen. 1993. High resolution differentiation of aeromonads. Med. Microbiol. Lett. 2:248-255.

25. Huys, G., I. Kersters, M. Vancanneyt, R. Coopman, P. Janssen, and K. Kersters. 1995. Diversity of Aeromonas sp. in Flemish drinking water production plants as determined by gas-liquid chromatographic analysis of cellular fatty acid methyl esters (FAMEs). J. Appl. Bacteriol, 78:445-455.

26. Huys, G., M. Vancanneyt, R. Coopman, P. Janssen, E. Falsen, M. Altwegg, and K. Kersters. 1994. Cellular fatty acid composition as a chemotaxonomic marker for the differentiation of phenospecies and hybridization groups in the genus Aeromonas. Int. J. Syst. Bacteriol. 44:651-658.

27. Janda, J. M. 1991. Recent advances in the study of the taxonomy, pathogenicity, and infectious syndromes associated with the genus Aeromonas. Clin. Microbiol. Rev. 4:397-410.

28. Janssen, P., R. Coopman, G. Huys, J. Swings, M. Bleeker, P. Vos, M. Zabeau, and K. Kersters. Evaluation of the DNA fingerprinting method AFLP as a new tool in bacterial taxonomy. Microbiology, in press.

29. Kämpfer, P., and M. Altwegg. 1992. Numerical classification and identification of Aeromonas genospecies. J. Appl. Bacteriol, 72:341-351.

30. Kuijper, E. J., A. G. Steigerwalt, B. S. C. I. M. Schoenmakers, M. F. Peeters, H. C. Zanen, and D. J. Brenner. 1989. Phenotypic characterization and DNA relatedness in human fecal isolates of Aeromonas species. J. Clin. Microbiol. 27:132-136.

31. Magee, J. T., E. A. Randle, S. J. Gray, and S. K. Jackson. 1993. Pyrolysis mass spectrometry characterisation and numerical taxonomy of Aeromonas spp. Antonie Leeuwenhoek 64:315-323.

32. Martinetti Lucchini, G., and M. Altwegg. 1992. rRNA gene restriction patterns as taxonomic tools for the genus Aeromonas. Int. J. Syst. Bacteriol. 42:384-389.

33. Martinez-Murcia, A. J., C. Esteve, E. Garay, and M. D. Collins. 1992. Aeromonas allosaccharophila sp. nov., a new mesophilic member of the genus Aeromonas. FEMS Microbiol. Lett. 91:199-206.

34. Millership, S. E., and S. V. Want. 1993. Characterisation of strains of Aero- 
monas spp. by phenotype and whole-cell protein fingerprint. J. Med. Microbiol. 39:107-113.

35. Miyata, M., T. Aoki, V. Inglis, T. Yoshida, and M. Endo. 1995. RAPD analysis of Aeromonas salmonicida and Aeromonas hydrophila. J. Appl. Bacteriol. 79:181-185.

36. Osborn, A. M., K. D. Bruce, P. Strike, and D. A. Ritchie. 1993. Polymerase chain reaction-restriction fragment length polymorphism analysis shows divergence among mer determinants from gram-negative soil bacteria indistinguishable by DNA-DNA hybridization. Appl. Environ. Microbiol. 59: 4024-4030.

37. Pitcher, D. G., N. A. Saunders, and R. J. Owen. 1989. Rapid extraction of bacterial genomic DNA with guanidium thiocyanate. Lett. Appl. Microbiol. 8:151-156.

38. Popoff, M. Y., C. Coynault, M. Kiredjian, and M. Lemelin. 1981. Polynucleotide sequence relatedness among motile Aeromonas species. Curr. Microbiol. 5:109-114.

39. Ramteke, P. W., S. P. Pathak, A. R. Gautam, and J. W. Bhattacherjee. 1993. Association of Aeromonas caviae with sewage pollution. J. Environ. Sci. Health Part A Environ. Sci. Eng. 28:859-870.

40. Russell, F. H. 1898 . An epidemic, septisemic disease among frogs due to the Bacillus hydrophilus fuscus. JAMA 80:1442-1449.

41. Schubert, R. H. W., M. Hegazi, and W. Wahlig. 1990. Aeromonas enterope- logenes species nova. Hyg. Med. 15:471-472.

42. Schubert, R. H. W., M. Hegazi, and W. Wahlig. 1990. Aeromonas ichthiosmia species nova. Hyg. Med. 15:477-479.

43. Seidler, R. J., D. A. Allen, H. Lockman, R. R. Colwell, S. W. Joseph, and O. P. Daily. 1980. Isolation, enumeration, and characterization of Aeromonas from polluted waters encountered in diving operations. Appl. Environ. Microbiol. 39:1010-1018.

44. Vos, P., R. Hogers, M. Bleeker, M. Reijans, T. van de Lee, M. Hornes, A. Frijters, J. Pot, J. Peleman, M. Kuiper, and M. Zabeau. 1995. AFLPTM: a new technique for DNA fingerprinting. Nucleic Acids Res. 23:4407-4414.

45. Warburton, D. W., J. K. McCormick, and B. Bowen. 1994. Survival and recovery of Aeromonas hydrophila in water: development of methodology for testing bottled water in Canada. Can. J. Microbiol. 40:145-148.

46. Wayne, L. G., D. J. Brenner, R. R. Colwell, P. A. D. Grimont, O. Kandler, M. I. Krichevsky, L. H. Moore, W. E. C. Moore, R. G. E. Murray, E. Stackebrandt, M. P. Starr, and H. G. Trüper. 1987. Report of the Ad Hoc Committee on Reconciliation of Approaches to Bacterial Systematics. Int. J. Syst. Bacteriol. 37:463-464.

47. Zabeau, M., and P. Vos. 1993. Selective restriction fragment amplification: a general method for DNA fingerprinting. Publication 0534858 A1. European Patent Office, Munich, Germany. 